\title{
Data Acquisition with 3DM Analyst Mine Mapping Suite
}

\author{
J. Birch ADAM Technology, Australia
}

\begin{abstract}
3DM Analyst Mine Mapping Suite is a digital photogrammetric software system that allows accurate threedimensional (3D) data to be generated in a wide range of environments. Recent developments have focussed on enhancing its ability to be used underground, for geological and structural mapping, as well as surveying and shotcrete thickness measurements.
\end{abstract}

Recent trials have demonstrated that surface models of underground mines, that are accurate to better than five mm root-mean-square (RMS), can be generated with less than 15 minutes of fieldwork and 10 minutes of processing. Although this level of accuracy is overkill for most applications, this allows shotcrete thickness to be quantified very accurately, in less time and at a fraction of the cost of a laser scanner, with the advantage that the same data can be immediately used for geological and structural mapping. Unlike using probe holes to assess thickness, the issues with taking point measurements to represent an area of the surface are avoided, as are the problems associated with the orientation of the probe holes and the creation of weak points that can initiate cracking of the shotcrete.

Because the same data can be used for such a wide range of applications, the need to physically inspect the heading at all is greatly reduced. Also, because the images form a permanent record, the ability of supervisors to inspect mapping for accuracy and completeness and for surface models to be revisited in light of new information can be invaluable.

The same tools that have been developed for shotcrete thickness measurement can also be used for monitoring slopes of open pit mines for movement, with accuracies at least as good as the best laser scanners used in mining today and over longer distances.

This paper presents the results of some of these recent trials and how they compare to the alternatives.

\section{Introduction}

Spray-on rock support systems, like shotcrete, have greatly increased safety in underground mining. However, because they obscure the rock mass behind the support system once they have been applied, they leave only a brief window of opportunity in the normal mining cycle for a geologist to inspect the rock face.

ADAM Technology's 3DM Analyst Mine Mapping Suite is a digital photogrammetric system that is able to capture and georeference essential geological and geotechnical information whilst introducing minimal delay to the normal mining cycle. The same data can also be used for surveying and engineering applications, including measuring overbreak and underbreak, convergence, and shotcrete thickness. This paper discusses some of the results that have been obtained in recent trials using the system for these applications, including objective measurements of the accuracy of the derived data.

\section{Operation}

3DM Analyst Mine Mapping Suite generates 3D data from sets of two-dimensional (2D) images. It takes 10-15 minutes of fieldwork to set up the lights, mark control points, and capture the necessary images, and about 10 minutes to process those images to generate a textured surface model consisting of between 100,000 and 2,000,000 points (depending on user settings) ready for analysis (Figure 1).

Georeferencing the data is typically accomplished by marking four control points on the walls in the supported area (Figure 2). Painting an identifier next to an existing gewi bolt head or plate is convenient, but a small dot can be painted instead if required. Surveying the control points can occur at any time and control points don't need to be used every round, provided images are captured every round and there is overlap 
between the previous round's images and the current round's images, which is normally the case. If the control points are marked up in areas that are unlikely to be damaged, then the surveyors can improve their efficiency by picking up control points for multiple rounds at one time. Thus, they would not have to visit the heading every round.

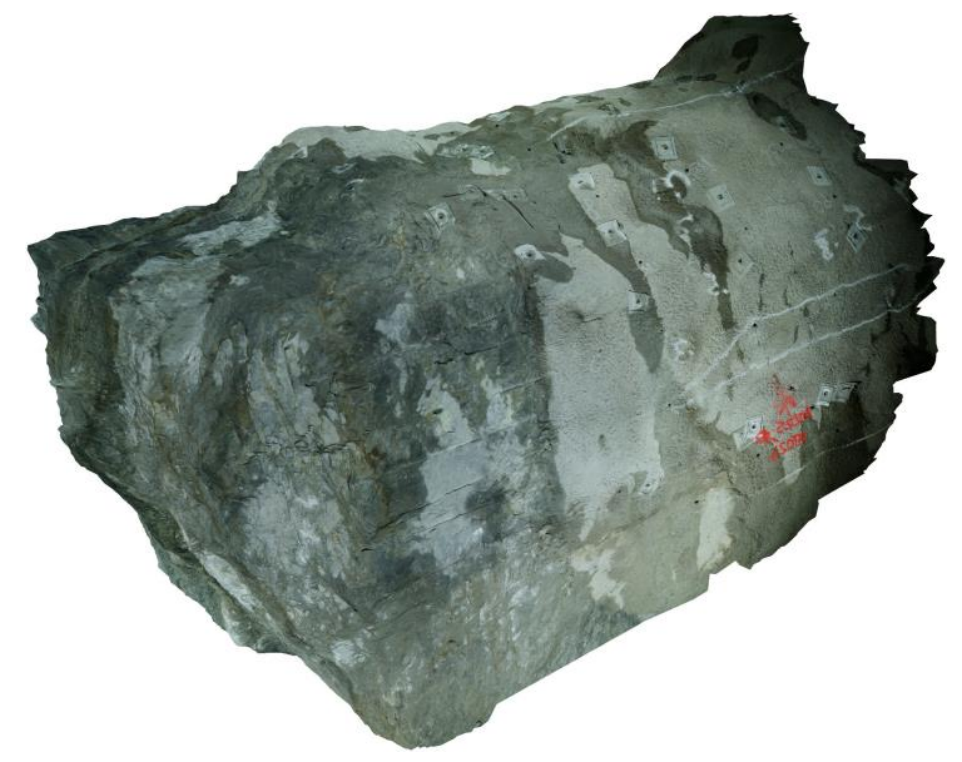

Figure 1 Digital terrain model (DTM) of a single round, seen from outside
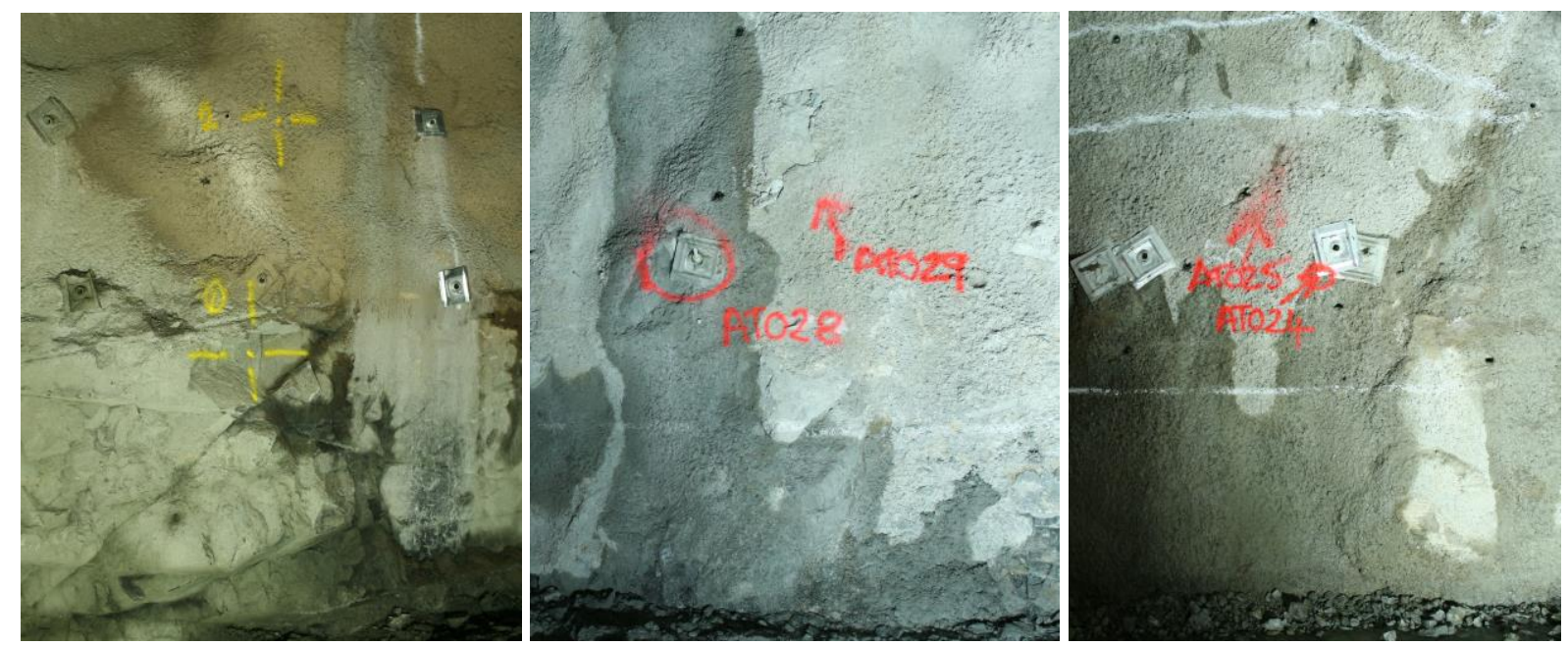

Figure 2 Control points painted on the wall, using existing gewi bolt heads and plate corners

The field equipment (Figure 3) includes a Canon EOS 5D digital SLR with a $20 \mathrm{~mm}$ lens and a panoramic camera mount with tribrach adapter that attaches to a standard set of survey legs. Three battery-operated LED lights provide even illumination with minimal shadowing and daylight colouring $(5500 \mathrm{~K})$ so the rock appears as it would on the surface, aiding identification. The lights are water resistant, can provide continuous illumination for four hours (enough for about 16 rounds) and take two hours to recharge.

To measure shotcrete thickness, the DTMs of two consecutive rounds are compared. Because the surface model not only includes the freshly-exposed rock face but also six metres or so of the supported area, it includes the area that was exposed rock in the previous round but has subsequently been shotcreted (Figure 4). The difference between the two DTMs in that area is therefore the shotcrete thickness (Figure 5). The colouring is user-selectable; in this case, red means a difference of less than $40 \mathrm{~mm}$ (underspray) while blue means a difference of more than $75 \mathrm{~mm}$ (overspray). The red area on the left of Figure 5 was already shotcreted in the previous round so the difference should be close to zero; the area on the right is freshly 
exposed, so the difference will be up to approximately $3.5 \mathrm{~m}$ in this case. The area in the middle was exposed rock in the previous round and shotcreted in the current round, so the difference between them gives the shotcrete thickness.
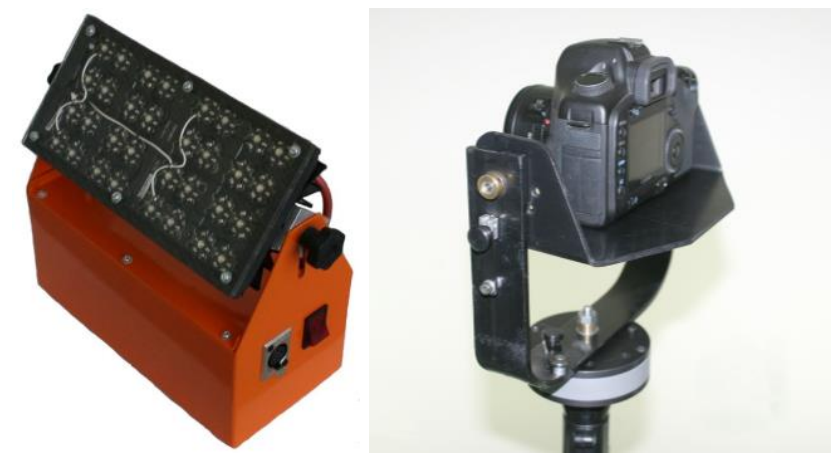

Figure 3 Light and panoramic camera mount

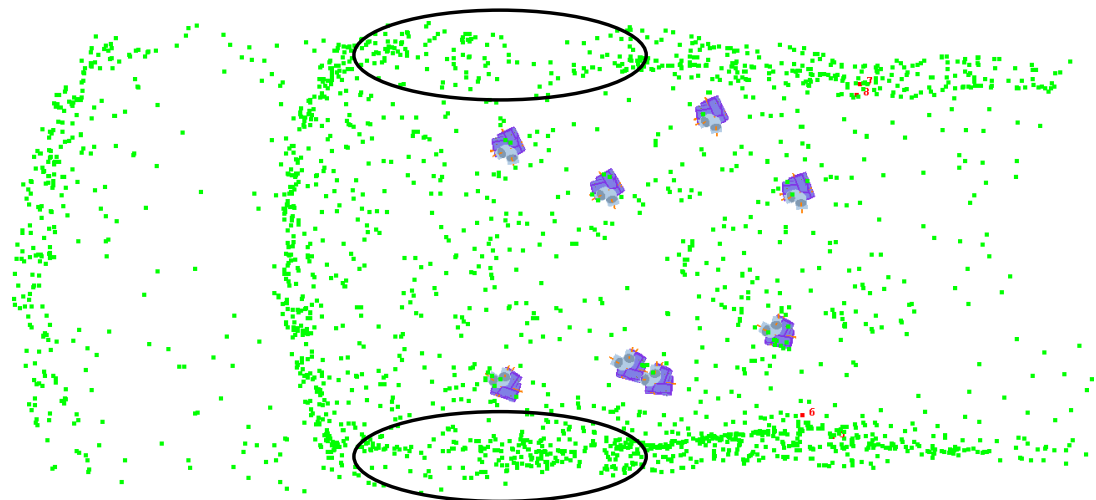

Figure 4 Overhead view of camera locations for two consecutive rounds with newly-shotcreted areas circled

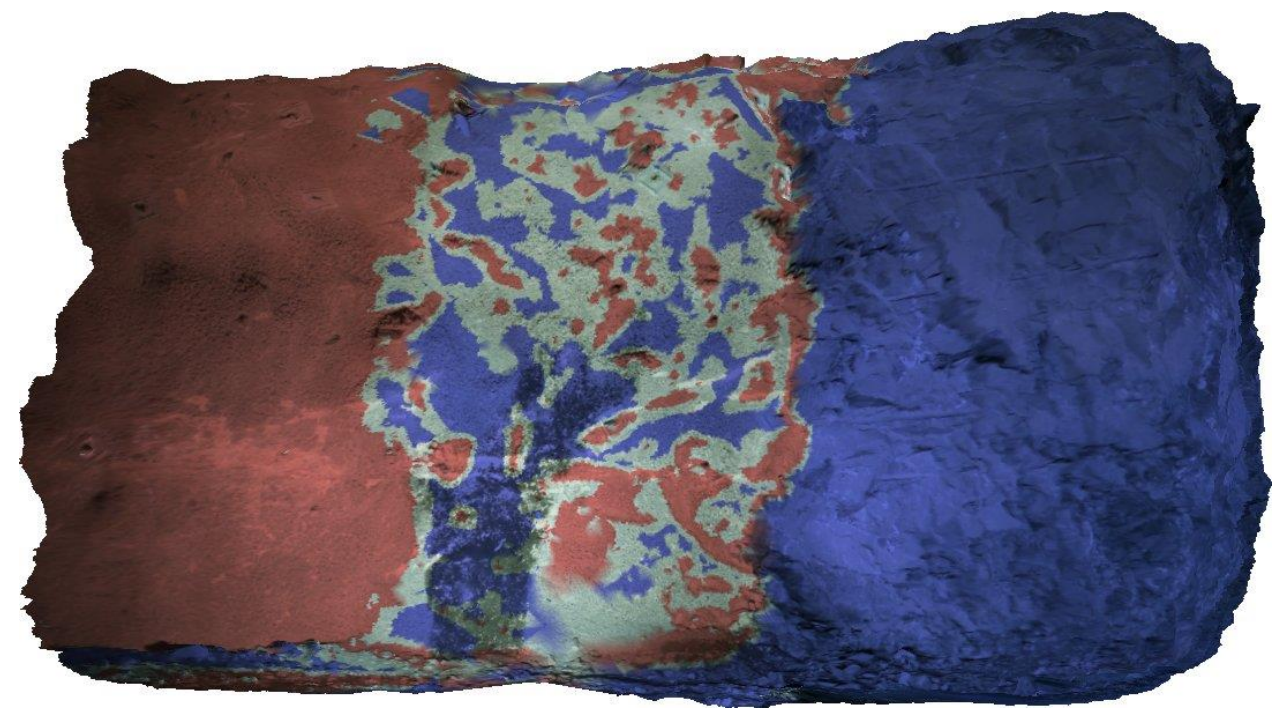

Figure 5 Colourised difference map of a DTM compared with the DTM from the previous round

For accurate shotcrete thickness measurement the images should be captured after hydroscaling, because otherwise the thickness measured will be less than it really is in areas where material was removed by the scaling. The desirability of accurate thickness measurements needs to be weighed against the cost of interrupting the shotcrete operator to capture the images. Unless detailed and accurate shotcrete thickness measurements are required every round it would be more efficient to capture post-hydroscaling images only 
occasionally and capture them prior to hydroscaling normally, where they can still be used for other purposes (like structural and geological mapping) without any problems.

\section{Methodology}

ADAM Technology participated in an onsite trial at Argyle Diamond mine (ADM) in the north of Western Australia from the 29-31 January 2008, to test the system's capabilities. Structural mapping and shotcrete thickness measurement capabilities were tested, as was the effect of capturing images before and after hydroscaling and the ability of the software to georeference a subsequent round without additional control points.

In each case the accuracy of the result was quantified, because knowing the accuracy is vital if decisions are to be made on the basis of the results. The normal approach in photogrammetry is to survey additional control points so that there is redundant information. The software will find the best fit for the data in a least squares sense; the differences between the derived control point locations and the original, surveyed locations - known as the residuals - give a good indication of the overall accuracy of the orientations.

Unfortunately, the residuals alone are not sufficient to determine the accuracy of the derived data. The orientation of discontinuities, for example, is determined by finding a best fit plane to a set of points digitised on the surface of the DTM. If those points are collinear, or if the error of those points is large with respect to their non-collinearity, then the orientation of the plane can be significantly affected. The shotcrete thickness measurements are also impacted by the density of the DTM points as well as their accuracy, because the surface is assumed to be piecewise planar (i.e. triangles are used to connect the points in the point cloud).

For this reason additional tests were conducted to verify the results. To quantify the mapping results the locations and orientations of a set of features were compared with the values derived by ADM's geologist. Shotcrete thickness measurements were quantified firstly, by comparing the thicknesses with the recorded probe hole depths and, secondly, by comparing the DTMs in areas that had not changed between rounds namely, the area that was already shotcreted in both rounds (e.g. the left third of Figure 5).

\section{Results}

\subsection{Structural mapping}

A set of features in various locations and orientations were digitised and compared with the values obtained by an ADM geologist (Figure 6). The orientations were all within the error range of the hand-mapped features. The orientation of some features, for example, the face on the right of Figure 6 and the traces on the active face, were likely to be far more accurate than the hand-mapped values due to the difficulty of aligning a clipboard with those features. The positional accuracy was also much higher. Several other advantages were also identified:

- At ADM, a geologist or geological technician is required to map every cut, spending about 20 minutes, in addition to the time taken to travel back and forth and to wait for access to the heading. With this system a relatively unskilled technician can capture the images and forward them to the surface for mapping, allowing the highly skilled personnel to spend more of their time utilising their unique skills analysing the data rather than driving around and waiting.

- With manual mapping, once mapping is complete there is no way to check the data or verify that nothing important has been overlooked, and variation between individuals cannot be assessed. Images, on the other hand, form a permanent record that can be revisited at any time (possibly in light of new information that only became available subsequently) and any mapping can be inspected by a supervisor to verify its accuracy and completeness. Mapping the same feature across multiple rounds to improve the accuracy of its orientation is also possible.

- Features can be digitised without regard to their distance from the supported area or their orientation. Trying to align a clipboard with a feature and then measuring the orientation of the clipboard is difficult to do accurately, especially when the feature presents itself as a flat face nearly perpendicular to the view direction. 


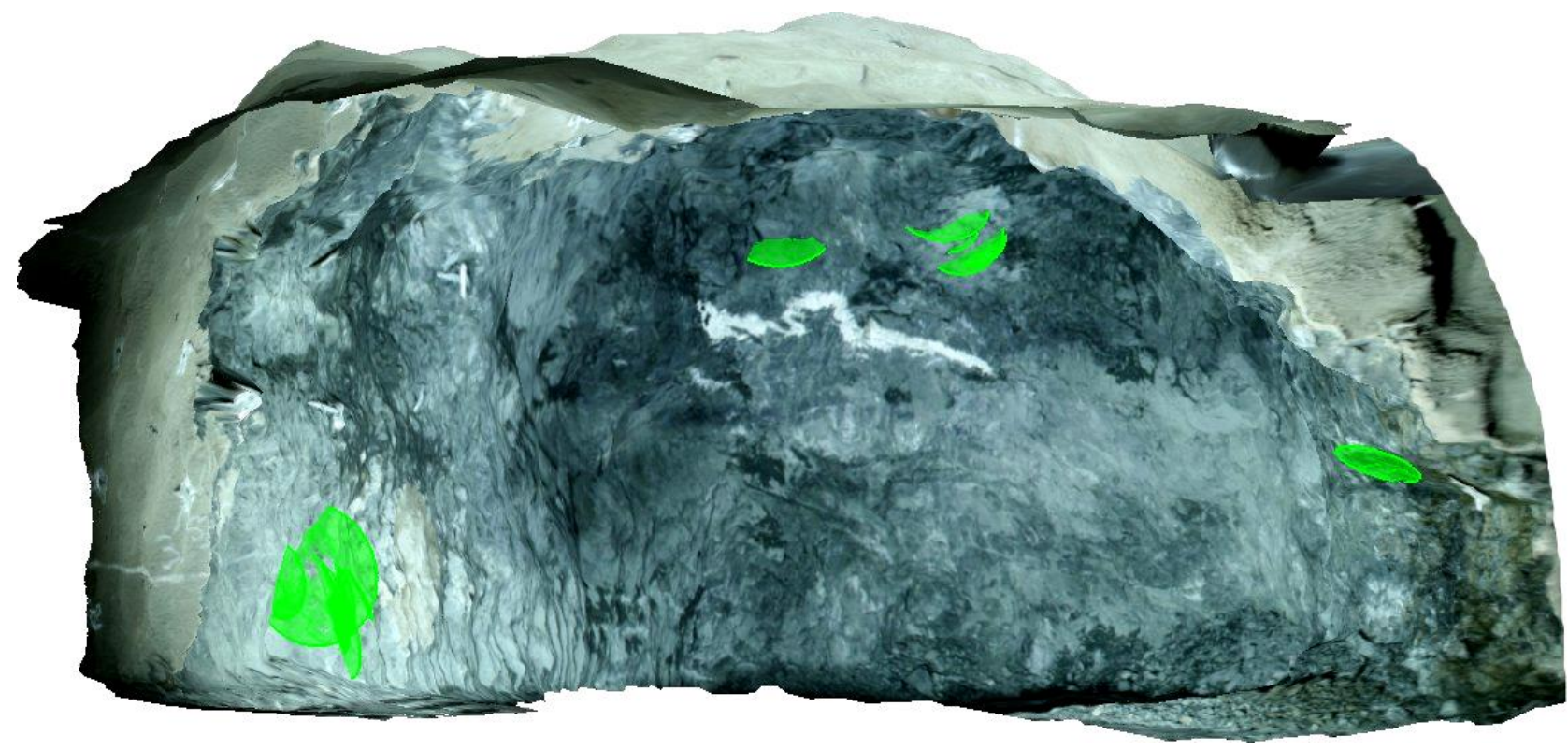

Figure 6 Features digitised for comparison in one of the headings

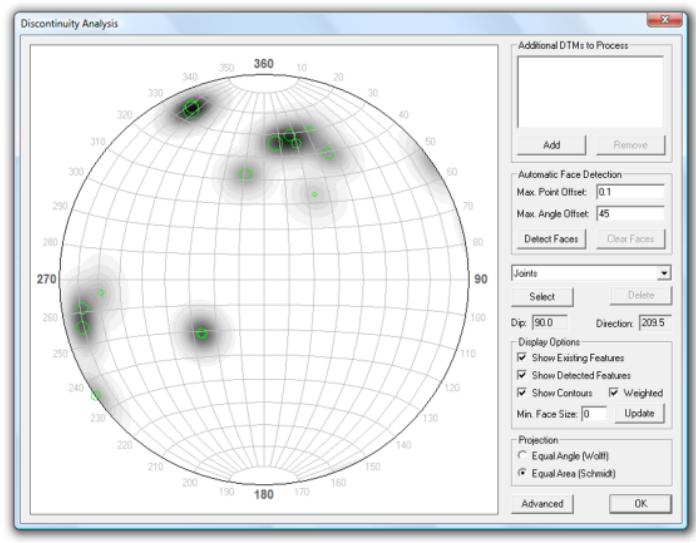

Figure 7 3DM analyst stereonet of the features shown in Figure 6

Table 1 Control point residuals for the heading shown in Figure 6

\begin{tabular}{|c|c|c|c|c|c|c|}
\hline \multirow{2}{*}{ Control Point ID } & \multicolumn{3}{|c|}{ Adjusted Data (m) } & \multicolumn{3}{|c|}{ Residuals (m) } \\
\hline & $X$ & $\mathrm{Y}$ & $\mathrm{Z}$ & $\mathrm{X}$ & Y & $\mathrm{Z}$ \\
\hline 1 & 30761.737 & 55882.573 & 9794.589 & -0.002 & 0.002 & 0.002 \\
\hline 2 & 30761.645 & 55882.516 & 9793.443 & -0.001 & 0.003 & 0.003 \\
\hline 3 & 30778.012 & 55887.569 & 9792.546 & 0.001 & 0.002 & 0.001 \\
\hline 4 & 30778.007 & 55887.715 & 9791.522 & 0.002 & 0.003 & 0.001 \\
\hline 111 (bolt on roof) & 30767.702 & 55888.122 & 9795.984 & 0.003 & -0.007 & -0.007 \\
\hline 112 (bolt on roof) & 30768.690 & 55887.732 & 9796.179 & -0.003 & -0.003 & -0.002 \\
\hline \multicolumn{4}{|c|}{ Control Point RMS (m) } & 0.002 & 0.004 & 0.004 \\
\hline \multicolumn{4}{|l|}{ Total RMS (m) } & & 0.006 & \\
\hline
\end{tabular}


Table 1 shows the control point residuals for this heading. The surveyors picked up a couple of bolt heads on the back for redundancy, capturing additional points like this is recommended because the marginal cost is low. They do not need to be marked in any way because as long as three control points can be identified by the user (such as those painted on the walls), the software can be asked to drive to the location of the remaining control points in the images for digitising.

\subsection{Shotcrete thickness}

Figure 5 shows the shotcrete thickness map for one side of one of the headings. Figure 8 shows a map in more detail, allowing the indicated probe hole depths to be compared with the values obtained by the software.
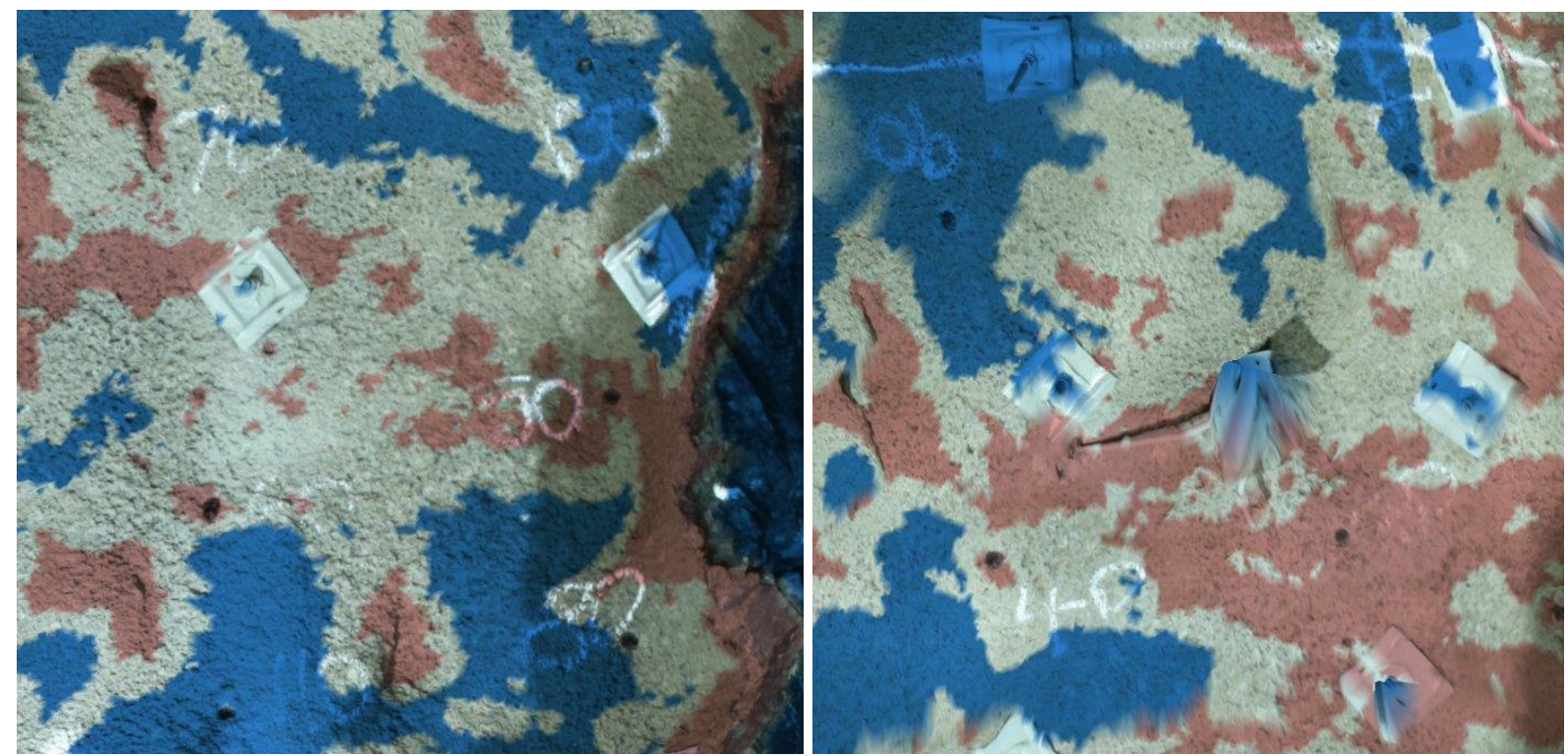

Figure 8 Detailed view of a DTM difference map showing indicated probe hole depths

While the agreement is reasonable overall, it is clear that, even with an extensive probe hole testing regime, the thickness of the shotcrete is so intricate and varied that it would be very difficult to obtain an adequate representation of its thickness without drilling so may probe holes that there would be very little shotcrete left. In some cases, large areas of overspray or underspray are completely missed, while in others, the hole has been very unlucky and managed to pick up an extremely localised and unrepresentative deviation in thickness (e.g. the lower-left measurement of "40" in the right-hand image of Figure 8). Because probe holes are also susceptible to errors introduced by the drilling angle and the possibility that the depth of the probe hole is not the same as the distance between the rock surface and the shotcrete surface, additional tests were conducted to establish the accuracy of the shotcrete thickness measurements: DTMs were compared in areas that should not have changed between rounds.

Figure 9 shows the result of the first such check, a 2.5 x $5.8 \mathrm{~m}$ area of shotcrete colourised yellow if the difference is $10-20 \mathrm{~mm}$ and red if the difference is in excess of $20 \mathrm{~mm}$. The RMS of the differences is $7.5 \mathrm{~mm}$. However, looking at the image it is clear that the wall has, in fact, changed - it was damaged by the bogger while the second round was being mucked out. Not only has the software correctly detected it, but it allows us to quantify it, the big gouge marked in red is over $20 \mathrm{~mm}$ deep, nearly half the nominal thickness of the shotcrete. Because of this the RMS figure is higher than it should be.

Checking the DTMs on the other side shows that the shotcrete has escaped relatively unscathed. In that case the RMS of the differences between the DTMs is $3.9 \mathrm{~mm}$, which seems to be sufficiently accurate to make definitive statements about the thickness of the shotcrete. Indeed, that is probably the limit of accuracy possible when it comes to shotcrete thickness measurements due to the rough nature of the surface.

Table 2 shows the control point residuals for the same heading, which agree well with the DTM accuracy determined previously. 
At another trial recently conducted in New South Wales a similar test was performed and the RMS of the DTM difference in the unchanged area was $3.7 \mathrm{~mm}$ in that case. The field technician who captured the images for that trial had only received a few hours of training at ADAM's offices yet managed to produce equivalent results performing the field work in the field for the first time and without assistance.

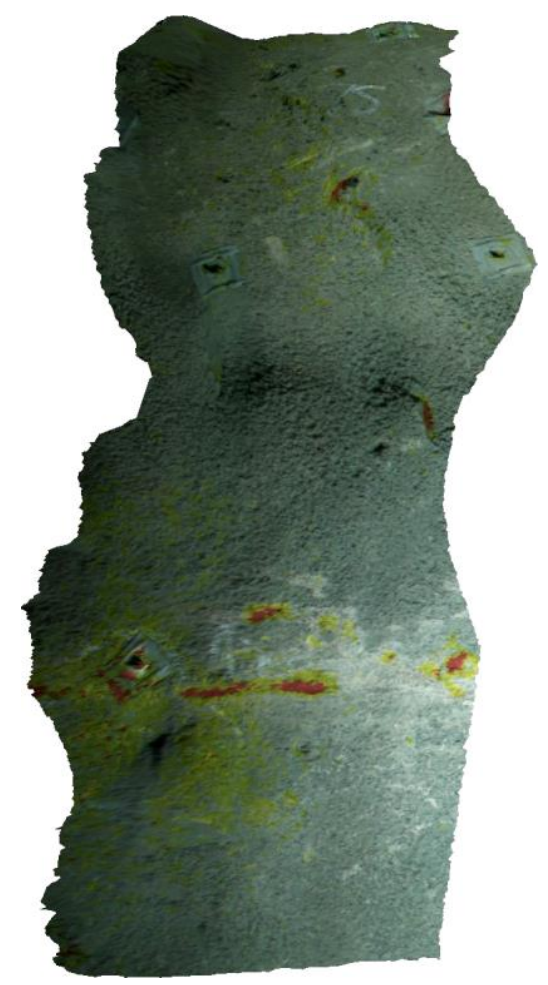

Figure 9 Accuracy check (note damage caused by bogger)

Table 2 Control point residuals

\begin{tabular}{ccccccc}
\hline \multirow{2}{*}{ Control Point Names } & \multicolumn{3}{c}{ Adjusted Data (m) } & \multicolumn{3}{c}{ Residuals (m) } \\
& $\mathrm{X}$ & $\mathrm{Y}$ & $\mathrm{Z}$ & $\mathrm{X}$ & $\mathrm{Y}$ & $\mathrm{Z}$ \\
\hline 5 & 30817.809 & 55870.939 & 9838.214 & 0.000 & 0.002 & -0.004 \\
6 & 30817.554 & 55871.350 & 9840.482 & 0.001 & -0.001 & 0.004 \\
7 & 30818.123 & 55875.586 & 9838.236 & 0.001 & -0.002 & 0.001 \\
8 & 30818.183 & 55875.539 & 9839.932 & -0.002 & 0.001 & -0.002 \\
\hline Control Point RMS (m) & & & & 0.001 & 0.002 & 0.004 \\
Total RMS (m) & & & & & 0.004 & \\
\hline
\end{tabular}

\subsection{Hydroscaling}

Figure 10 shows the effect of hydroscaling on the surface. Yellow means a difference of $15-30 \mathrm{~mm}$ in this case, and red a difference in excess of $30 \mathrm{~mm}$. The nominal shotcrete thickness for this heading is $50 \mathrm{~mm}$; these differences are therefore significant and must be taken into account when analysing the DTM differences where images were captured prior to scaling, because they can make the shotcrete appear thinner than it really is. On the other hand, it never gives a false sense of security by making the shotcrete appear thicker than it really is.

If the production implications of capturing images after scaling prevent the fieldwork from being inserted at that point in the cycle on a regular basis, it might still be worth occasionally capturing images after scaling as a QA check. Capturing images prior to scaling does not affect their ability to be used for structural mapping. 


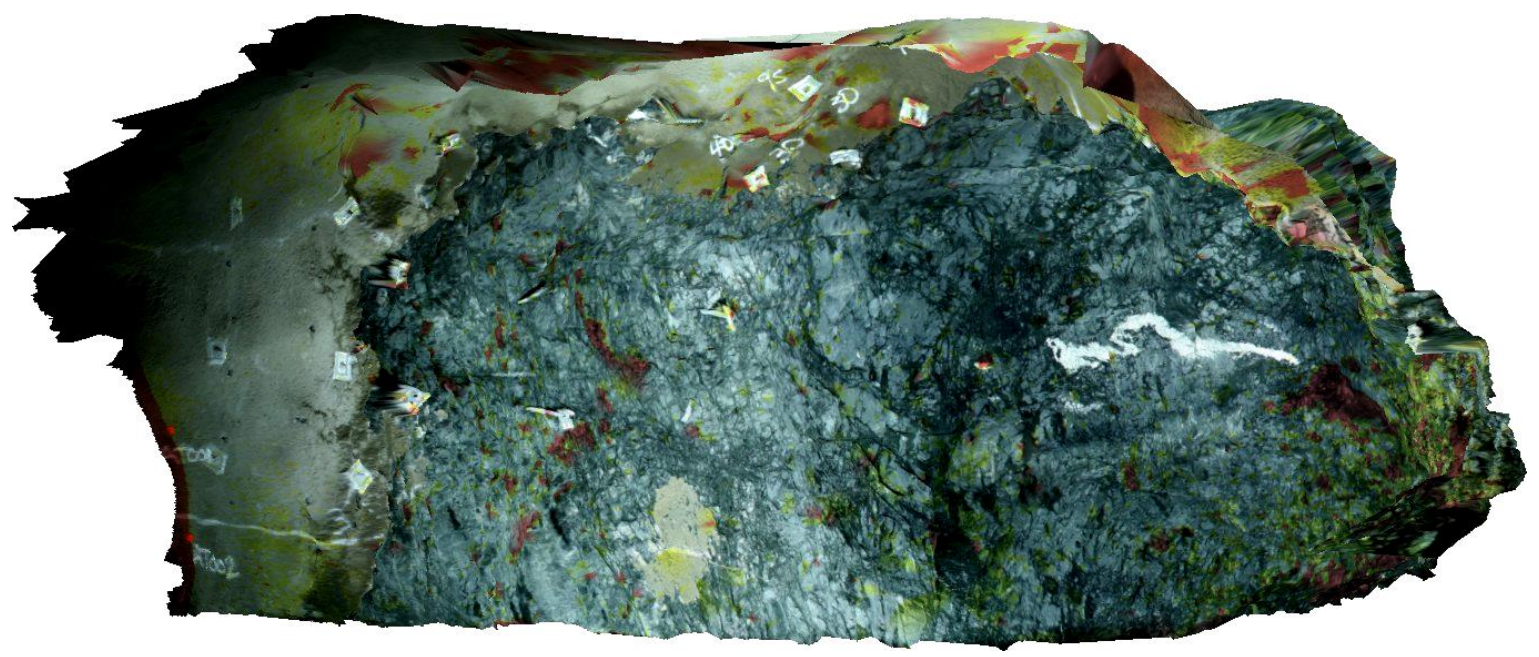

Figure 10 The effect of hydroscaling

\subsection{Minimising control}

During the three day trial a total of six headings and a pumping station chamber were captured. Only two headings could be captured more than once due to scheduling issues, and only one of those was of conventional geometry where the second round of images could be georeferenced using the first round. Figure 5 shows the shotcrete thickness measured when that heading was georeferenced by digitising the same control points in the second set of images. Figure 11 shows the same heading georeferenced entirely by the images captured in the previous round. Close inspection of the two difference maps shows some minor differences but certainly nothing that would impact on a user's ability to make correct decisions.

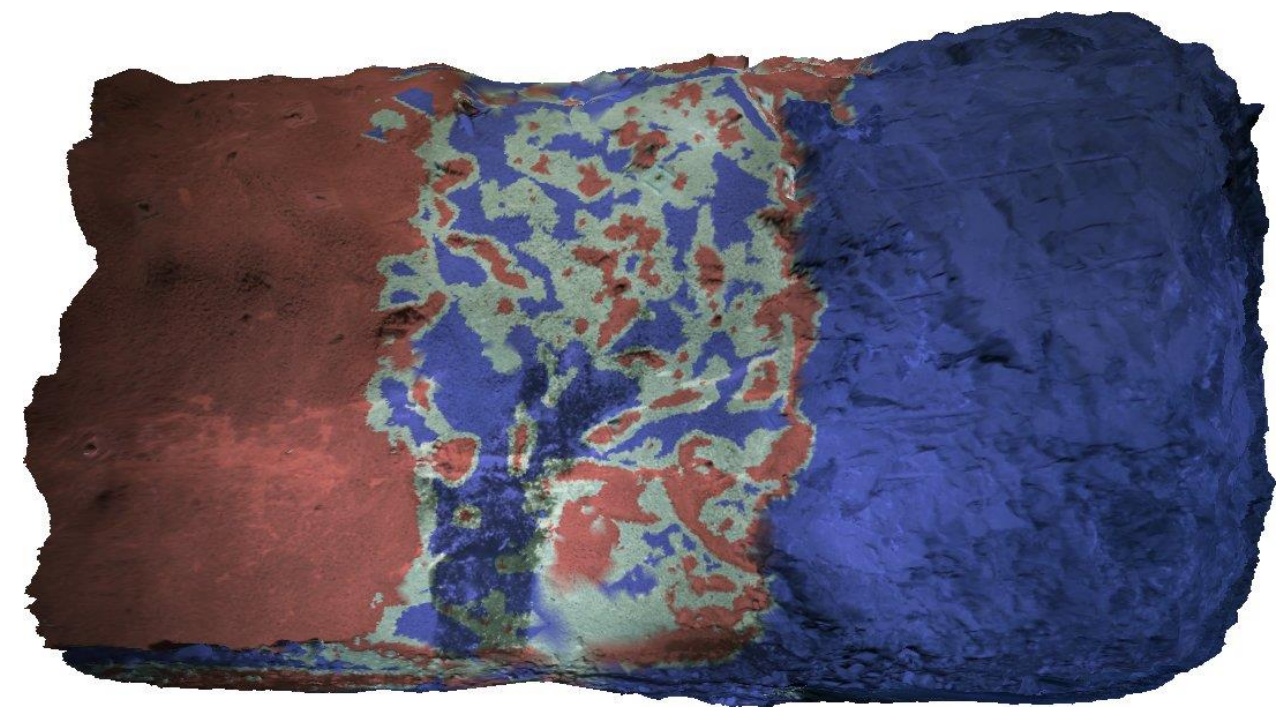

Figure 11 Shotcrete thickness measured without using control points for the second round

\subsection{Error detection}

One final point worth noting with this trial was that the control point information shown in Table 2 is not actually the data that was supplied by the ADM surveyors. The original data is reproduced in Table 3 .

When this data was used, the software reported large vertical residuals on both sides, leading to some confusion as to whether the correct points were being digitised in the images.

A quick check of the distances between the points on each side as supplied by the surveyors, and the points as digitised in the images, showed that the distances matched up very well, if the points swapped sides. 
Table 3 Original control point data for the heading shown in Table 2

\begin{tabular}{ccccc}
\hline No. & $\mathrm{X}(\mathrm{m})$ & $\mathrm{Y}(\mathrm{m})$ & $\mathrm{Z}(\mathrm{m})$ & Name \\
\hline 1 & 30817.809 & 55870.937 & 9838.218 & AT007 \\
2 & 30817.553 & 55871.351 & 9840.478 & AT008 \\
3 & 30818.122 & 55875.588 & 9838.235 & AT005 \\
4 & 30818.185 & 55875.538 & 9839.934 & AT006 \\
\hline
\end{tabular}

In other words, the points labelled 5 and 6 in the survey data were actually points 7 and 8 painted on the walls (and digitised in the images), and vice-versa. Renumbering the points in the control point file immediately solved the problem and generated the residuals shown in Table 2.

This highlighted a very important feature of the software, i.e. it is very difficult to make a mistake at any point in the process and not have the software detect it. It is very easy to make this kind of mistake in the field, so users should expect to get bad data from time-to-time, but that does not mean the end result will be incorrect. The software is perfectly capable of detecting when there is a problem and, giving the user the tools they need to figure out what that problem is, before proceeding. This is critically important when there are people downstream who are relying on the data being generated.

\subsection{Other applications}

During the trial ADM's surveyors asked if a pumping station chamber could be modelled for checking against design to ensure it was large enough (Figure 12). The chamber was larger than the headings mapped until that point ( $10 \mathrm{~m}$ wide by $10 \mathrm{~m}$ high; the area captured for the trial was $30 \mathrm{~m}$ long) but the fieldwork was similar. The actual photography took 10 minutes to capture 18 images and processing took 22 minutes from start to finish. The software's ability to generate cross-sections at a user-specified interval, direction and declination was used to create data for loading into Vulcan. This same functionality can be used for measuring overbreak and underbreak and checking headings against design; greatly increasing the amount and quality of the data the surveyors can generate while simultaneously reducing the effort required to generate them. As an additional benefit, this data is generated from exactly the same DTMs that are used for mapping and shotcrete thickness measurement at no additional cost.

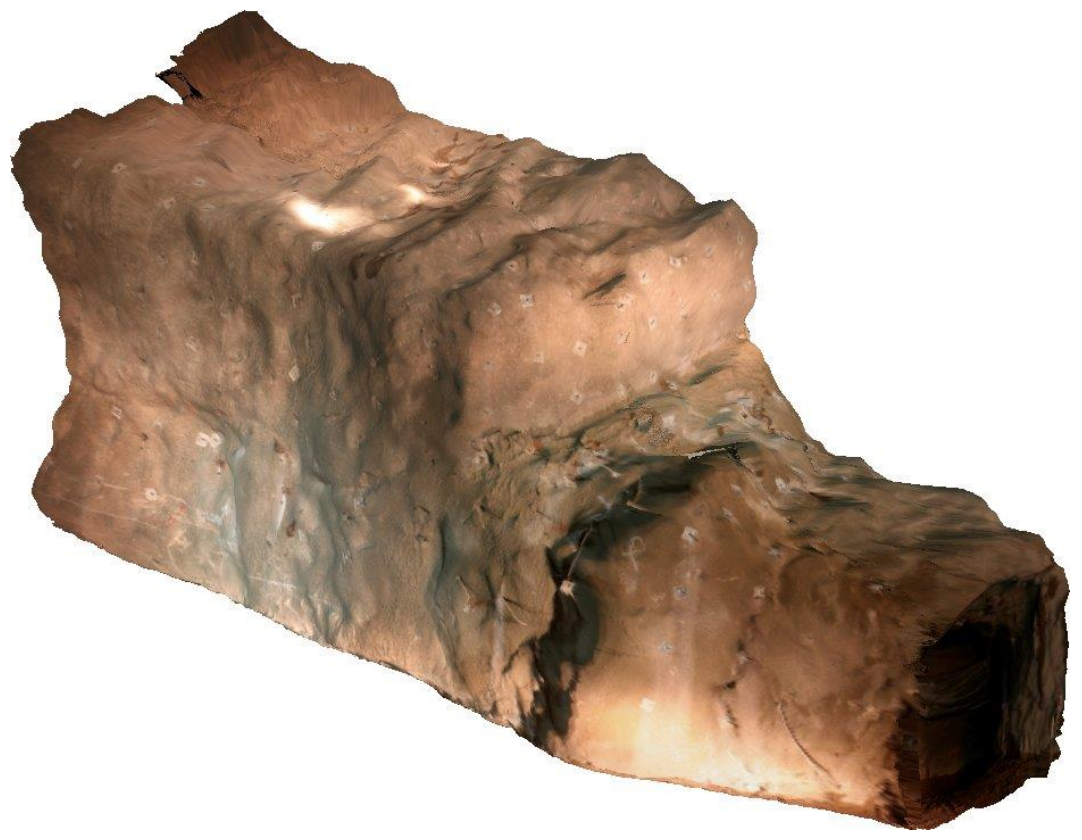

Figure 12 Pumping station chamber 


\section{Conclusion}

The trial results indicate that not only can 3DM Analyst Mine Mapping Suite perform all of the tasks expected, but can do so more accurately and with less effort than any of those tasks require using current techniques. The system also includes mechanisms that can be used to predict the accuracy of the results as well as automatically detect errors in the data being given to it.

Apart from in-cycle production use, the system could also be valuable as a training tool for shotcrete operators, giving them feedback on their performance.

\section{Acknowledgements}

ADAM Technology would like to thank Argyle Diamonds and Macmahon's Special Underground Drilling and Ground Support Services for the use of the data presented in this paper and their assistance and support with this trial. 